\title{
Preparation and characterization of sodium dodecyl sulfate doped polypyrrole solid phase micro extraction fiber and its application to endocrine disruptor pesticide analysis
}

\author{
Korcan Korba ${ }^{a}$, Levent Pelit ${ }^{\mathrm{a}, *}$, Füsun Okçu Pelit ${ }^{\mathrm{a}}$, K. Volkan Özdokur ${ }^{\mathrm{a}}$, Hasan Ertaş ${ }^{\mathrm{a}}$, \\ Ahmet E. Eroğlu ${ }^{b}$, F. Nil Ertaş ${ }^{a}$ \\ a Ege University, Science Faculty, Department of Chemistry, Bornova 35100, İmir, Turkey \\ ${ }^{\mathrm{b}}$ Department of Chemistry, Izmir Institute of Technology, Urla 35430, Izmir, Turkey
}

\section{A R T I C L E I N F O}

\section{Article history:}

Received 31 January 2013

Accepted 13 April 2013

Available online 20 April 2013

\section{Keywords:}

Polypyrrole fiber

Head space solid phase micro extraction

Endocrine disruptor pesticide

Gas chromatography

Red wine

\begin{abstract}
A B S T R A C T
A robust in house solid-phase micro extraction (SPME) surface has been developed for the headspace (HS)-SPME determination of endocrine disruptor pesticides, namely, Chlorpyrifos, Penconazole, Procymidone, Bromopropylate and Lambda-Cyhalothrin in wine sample by using sodium dodecylsulfate doped polypyrrole SPME fiber. Pyrrole monomer was electrochemically polymerized on a stainless steel wire in laboratory conditions in virtue of diminishing the cost and enhancing the analyte retention on its surface to exert better selectivity and hence the developed polymerized surface could offer to analyst to exploit it as a fiber in headspace SPME analysis. The parameters, mainly, adsorption temperature and time, desorption temperature, stirring rate and salt amount were optimized to be as $70^{\circ} \mathrm{C}$ and $45 \mathrm{~min}, 200{ }^{\circ} \mathrm{C}, 600 \mathrm{rpm}$ and $10 \mathrm{~g} \mathrm{~L}^{-1}$, respectively. Limit of detection was estimated in the range of $0.073-1.659 \mathrm{ng} \mathrm{mL}^{-1}$ for the pesticides studied. The developed method was applied in to red wine sample with acceptable recovery values (92-107\%) which were obtained for these selected pesticides.
\end{abstract}

(C) 2013 Elsevier B.V. All rights reserved.

\section{Introduction}

One of the major challenges in analytical chemistry is to develop faster, easier and reliable methods for the quantification of the analyte within acceptable accuracy. Growing demand for greener and faster preconcentration techniques has resulted in intensive research on alternative extraction methods. Solid phase micro extraction (SPME) is a simple, solvent free, fast and inexpensive, technique for attaining very low limit of detection for trace organic compounds [1]. The extracting phase is a thin polymer film coated fiber and the analyte preconcentrated on the fiber is, then, rapidly delivered to for example a GC column [2]. SPME can also be combined with headspace technique (HS-SPME) for the determination of volatile organic compounds eliminating the interferences from complex matrices [3,4] and the life time of fiber is increased.

Several kinds of SPME fibers are commercially available including polydimethylsiloxane (PDMS) [5-11], carbowax-divinlybenzene (CW-DVB) [12,13], polydimethylsiloxane-divinlybenzene (PDMS-DVB) [14-16], polyacrylate (PA) [17-19], divinylbenzene-carboxen-polydimethylsiloxane (DVB-CAR-PDMS) [20-22]. In recent years, studies have been

\footnotetext{
* Corresponding author. Tel.: +90 232 3112389; fax: +90 2323888264 .

E-mail address: levent.pelit@ege.edu.tr (L. Pelit).
}

focused on fabricating low cost, simple, robust and long-life fiber for the target analyte [23]. A variety of methods for the production of fibers have been developed such as sol-gel [24], and physical and electrochemical polymerization [25]. Among these, electrochemical polymerization is the method of choice as the film thickness can be controlled easily and polymers with different functional groups can be formed conveniently by changing dopant ions or using substituted monomers under controlled electrochemical conditions [26].

Polypyrrole (PPy) is one of the most studied conducting polymers due to its environmental stability, relative ease of synthesis and higher conductivity than many other polymers [27]. Thermal and mechanical stability of PPy films could be greatly influenced by parameters such as type of the dopant ion, electrochemical conditions and reaction medium used [28]. The electrochemical polymerization in aqueous solutions has offered the possibility for a large number of anions to be applied as the dopant which determines the chemical characteristics of PPy films [29,30]. PPy was also synthesized in the presence of different anionic surfactants [31-34].

It was reported earlier that the fibers coated with dodecylsulfate-doped polypyrrole (PPy-DS) were stable in high temperatures (up to $300^{\circ} \mathrm{C}$ ) and good adhesive features of film was observed. It is evident that the lower thermal stability of the film restricts the range of applications associated with this film 
as an SPME fiber. Moreover, the polymer film can be coated on a metal wire which displays better mechanical strength than silica fibers. Various support materials were used for fabricating SPME fibers including platinum [35,36], gold [37], stainless steel [38-41], gold microarray electrode [42], and multiwalled carbon nanotubes [43].

The main objective of this study is to fabricate a PPy coated SPME fiber for volatile pesticide residue analysis. PPy fiber was coated electrochemically on a stainless steel wire by using cyclic voltammetry (CV). The surface of the fiber was characterized by using scanning electron microscopy (SEM), Fourier transform infrared spectroscopy (FT-IR) and thermogravimetric analysis (TGA) techniques. The performance of the SPME fiber prepared was evaluated upon analyzing a number of volatile pesticide residues by headspace technique in red wine sample.

There is a worldwide trend toward setting specific, lower maximum residue limits (MRL) for pesticide residues in wine, which would range from 0.01 to $2 \mathrm{mg} / \mathrm{kg}$ for different pesticides [44]. Sensitive and selective methods are therefore required for their reliable detection. Most of SPME methods developed to date for pesticide analysis in wine matrix are usually used by direct immersion technique $[45,46]$. A few studies have been published for the determination of one or two pesticide residues in wine samples by HS-SPME $[47,48]$. This study is the first attempt to make simultaneous determination of five endocrine disruptor pesticides [49-51] in wine samples commonly used in grapevine control in Turkey such as, Chlorpyrifos (CP), Penconazole (PNZ), Procymidone (PRC), Bromopropylate (BRP) and Lambda-Cyhalothrin (LMD) by HS-SPME method.

\section{Experimental}

\subsection{Chemicals and reagents}

All reagents were of analytical reagent grade. Aqueous solutions were prepared with ultra pure water $\left(18.2 \mathrm{M} \Omega \mathrm{cm}^{-1}\right)$ from a MilliPore Milli-Q Gradient water purification system. Pyrrole, (Alfa-Aesar, 98\%), was purified by distillation under argon at atmospheric pressure before use. Pesticides standards; Aldrin $(2,3,4,10,10$-Hexachloro-1,4,4a,5,8,8a-hexahydro-1,4:5,8dimethanonaphthalene) (97\%), Chlorpyrifos (O,O-Diethyl O-3,5,6-trichloropyridin-2- phosphorothioate) (98.4\%), Penconazole (1-[2-(2,4-dichloro phenyl)-n-pentyl]-1H-1,2,4-triazole) (99.2\%), Bromopropylate (isopropyl-4,4'-dibromobenzilate) (97.5\%), Procymidone (3-(3,5-dichlorophenyl)-1,5dimethyl-3-azabicyclo[3.1.0]hexane-2,4-dione) (98\%), and Lambda-cyhalothrin (3-(2-chloro-3,3,3-trifluoro-1-propenyl)2,2-dimethyl-cyano (3-phenoxy phenyl) methyl cyclopropane carboxylate) (98.5\%) were purchased from Dr. Ehrenstarfer $\mathrm{GmbH}$. A stock solution of these pesticides was prepared in ethanol and stored at $4{ }^{\circ} \mathrm{C}$ before use.

The wine sample examined for the study was obtained commercially from the local market. The red wine sample was purchased from a local village, September 2006, with an alcohol percentage of $12 \%$. The wine was stored at $4{ }^{\circ} \mathrm{C}$ before the analysis.

\subsection{Instrumentation}

SPME fiber coating was carried out using a PalmSence Electrochemical Interface. A three-electrode system consisted of a stainless steel wire (316 type, i.d. $0.3 \mathrm{~mm}$, working electrode), a platinum wire (auxiliary) electrode and an $\mathrm{Ag} / \mathrm{AgCl}$ electrode as reference electrode was used for all cyclic voltammetric measurements. Functional groups of PPy-DS were identified by Perkin-Elmer Pyris FTIR spectrophotometer. Thermal decomposition study of PPy-DS was carried out by using PerkinElmer Diamond TG/DTA analyzer. Philips XL30 SFEG was used for SEM measurements. The SPME holder for manual sampling was obtained from Supelco (Bellefonte, PA, USA). A heating magnetic stirrer, purchased from IKA-RCT (Germany) was used with a $10 \mathrm{~mm}$ stirring bar. Heraeus RT500 stove was used for fiber conditioning. Transsonic 460/H was used for ultrasonic treatments.

GC analyses were performed with a gas chromatograph from Agilent Model 6890 Series, equipped with 7673A auto sampler and HP ECD detector systems. A DB-1 fused silica column $(30 \mathrm{~m} \times 0.32 \mathrm{~mm}$ I.D. and film thickness $0.25 \mu \mathrm{m}$ ) was employed. Helium and nitrogen (99.999\%) were used as carrier and make-up gas, respectively.

The injection port and detector were operated at $200^{\circ} \mathrm{C}$ and $300^{\circ} \mathrm{C}$, respectively. The fiber was introduced into the chromatographic columns using splitless mode injection. SPME fiber inserted to GC injection port and GC analysis was started manually. The GC split valve was closed for $5 \mathrm{~min}$, and helium was used as carrier gas with a flow rate of $40.0 \mathrm{~mL} \mathrm{~min}^{-1}$. The flow rate of carrier gas was adjusted at $1.5 \mathrm{~mL} \mathrm{~min}^{-1}$. The separation of pesticides on GCECD was performed by a temperature program as follows: $50^{\circ} \mathrm{C}$ for $2 \mathrm{~min}$ increased to $150^{\circ} \mathrm{C}$ at a rate of $10^{\circ} \mathrm{C} \mathrm{min}^{-1}$ and increased to $200^{\circ} \mathrm{C}$ at a rate of $2{ }^{\circ} \mathrm{C} \mathrm{min}^{-1}$ and increased to $280^{\circ} \mathrm{C}$ at a rate of $8{ }^{\circ} \mathrm{Cmin}^{-1}$. After GC analysis the fiber was inserted to GC injection port for $15 \mathrm{~min}$ to remove retained pesticide from the fiber surface.

\subsection{Fiber coating and conditioning}

PPy-DS film was prepared electrochemically according to the procedure as given elsewhere for platinum wire [31]. PPy-DS film was directly electrodeposited on the surface of a stainless steel wire electrode from an aqueous solution containing $0.1 \mathrm{M}$ pyrrole and $7.0 \times 10^{-3} \mathrm{M}$ SDS by using cyclic voltammetry. Prior to the electropolymerization, the steel wire was immersed in acetone in a test tube and placed in an ultrasonic bath for $15 \mathrm{~min}$ and subsequently, washed with distilled water. After purging the SDS solution with nitrogen gas for five min, the electropolymerization procedure was initiated by cycling the potential in a range of $0.5-1.2 \mathrm{~V}$ with a scan rate of $20 \mathrm{mV} \mathrm{s}^{-1}$ for a background recording. Upon addition of pyrrole to the solution, electrooxidation signal of pyrrole at $0.84 \mathrm{~V}$ has increased on successive cycling indicating the electropolymerization. Electrochemical coating procedure was completed after 10 cycles of the potential.

The metal wire coated with PPy-DS film was washed with methanol, acetone, and water in sequence respectively, and dried under nitrogen gas flow. Then, it was heated in an oven at $100^{\circ} \mathrm{C}$ for $20 \mathrm{~min}$ and connected to SPME holder. Before use, the fiber was conditioned at $200^{\circ} \mathrm{C}$ in a GC injection port under helium gas for an hour till a clear blank sample was obtained.

\subsection{Characterization of SPME fiber}

The film thickness and surface morphology of the SPME fiber developed were characterized by using the SEM recordings. The surface of the PPy-coated stainless steel wire was cut into a $2 \mathrm{~cm}$ long piece and then, analyzed on a SEM $(7.0 \mathrm{kV}$ accelerating potential) with different magnification. Thermal stability of the fiber was analyzed by TGA by using $5 \mathrm{mg}$ of sample in air flow $\left(100 \mathrm{~mL} \mathrm{~min}^{-1}\right)$ at a heating rate of $10^{\circ} \mathrm{C} \mathrm{min}^{-1}$. FTIR spectra of PPy-DS film in $\mathrm{KBr}$ pellets were recorded in the range of $450-4000 \mathrm{~cm}^{-1}$ with a $2 \mathrm{~cm}^{-1}$ spectral resolution. Two hundred scans were recorded for each FTIR spectrum. 


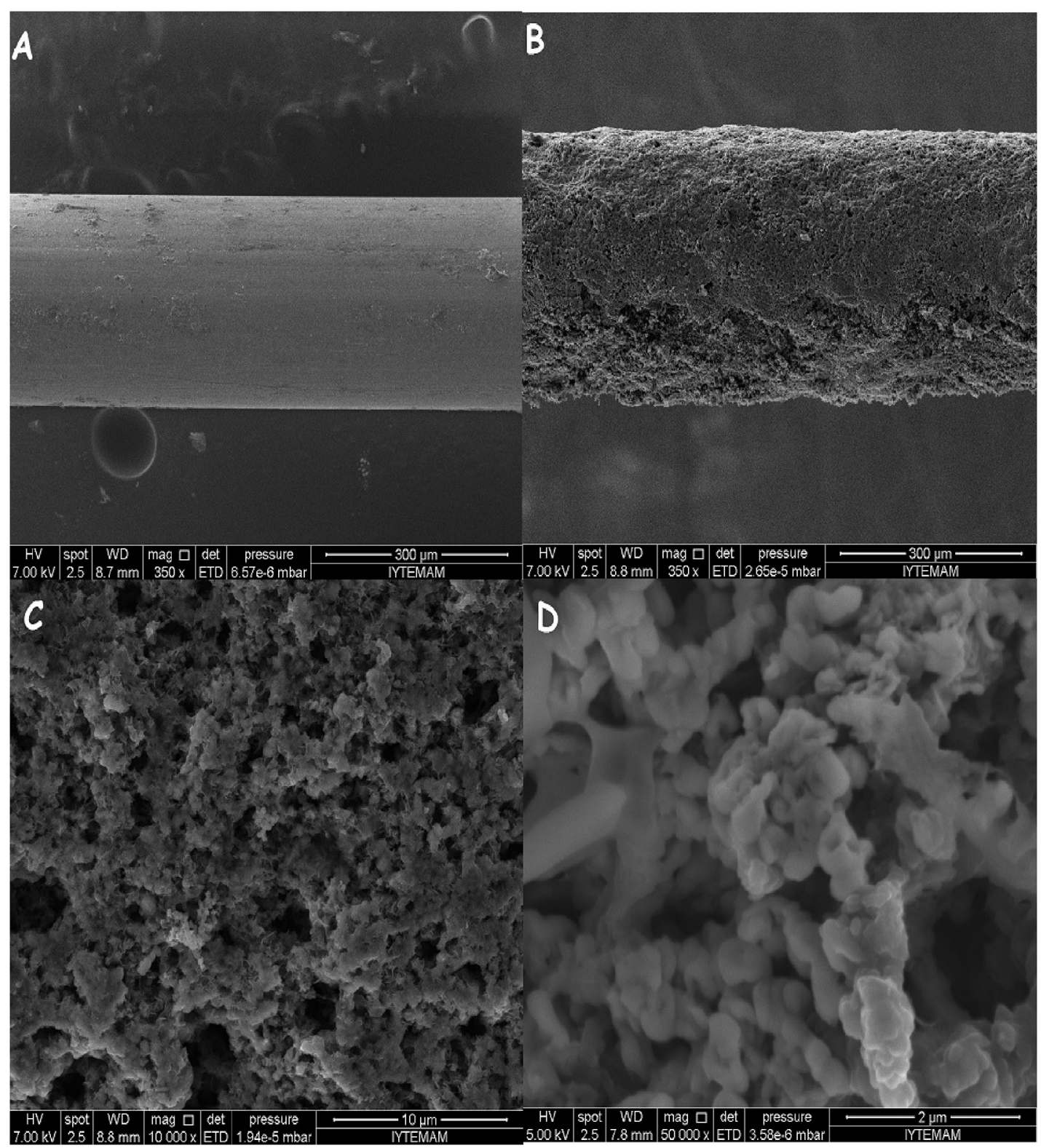

Fig. 1. SEM images of (A) bare stainless steel wire and (B) PPy-DS film at 350, (C) PPy-DS film at 10000 and (D) 50,000 folds magnification.

\subsection{The procedure for HS-SPME measurements}

The extraction ability of the prepared PPy-DS fibers in head space mode was evaluated by using aqueous solutions spiked with six organochlorine pesticides including aldrin as an internal standard. A $10.0 \mathrm{~mL}$ of standard pesticide mixed solution prepared in $12.0 \%$ ethanol solution was placed in a $20.0 \mathrm{~mL}$ glass vial with a PTFE-silicon septum. Similarly, $10.0 \mathrm{~mL}$ of red wine samples were spiked with target pesticide standards. After the addition of sodium chloride and magnetic stirring bar, the vial was tightly sealed with an aluminum cap to prevent sample loss due to the evaporation. The vial was placed on a hot plate set at $70^{\circ} \mathrm{C}$ and encircled with two pieces of round shaped Teflon for isolation. The mixture was allowed to reach the equilibrium for $5 \mathrm{~min}$ and then, the fiber housed in manual SPME holder was inserted into the vial at a fixed height above the solution. After a predetermined time, the fiber was withdrawn into the needle and immediately introduced into the injection port of the GC for $5 \mathrm{~min}$.

\section{Results and discussion}

\subsection{Polymer film preparation and characterization}

Since the creation of a uniform and stable coating on the SPME fiber is essential for the precision and accuracy, electropolymerization is often the method of choice as the thickness of the fiber can be controlled by the number of CV cycles. In addition to the film thickness, evenly distributed porous structure has also a paramount effect on the head space adsorption process as it provides higher extraction efficiency compared with non-porous films. The SEM images of the fiber depicts that PPy-DS films posses a well distributed porous structure (Fig. 1 ).

The thickness of the fiber coating can be controlled by tailoring the electrochemical conditions. The thickness of the PPy-DS coating under these conditions was estimated as $40 \pm 2 \mu \mathrm{m}$ from SEM images.

Fig. 2 displays the thermogravimetric results of the coated film. The weight loss in the temperature range of $30-80^{\circ} \mathrm{C}$ can be 


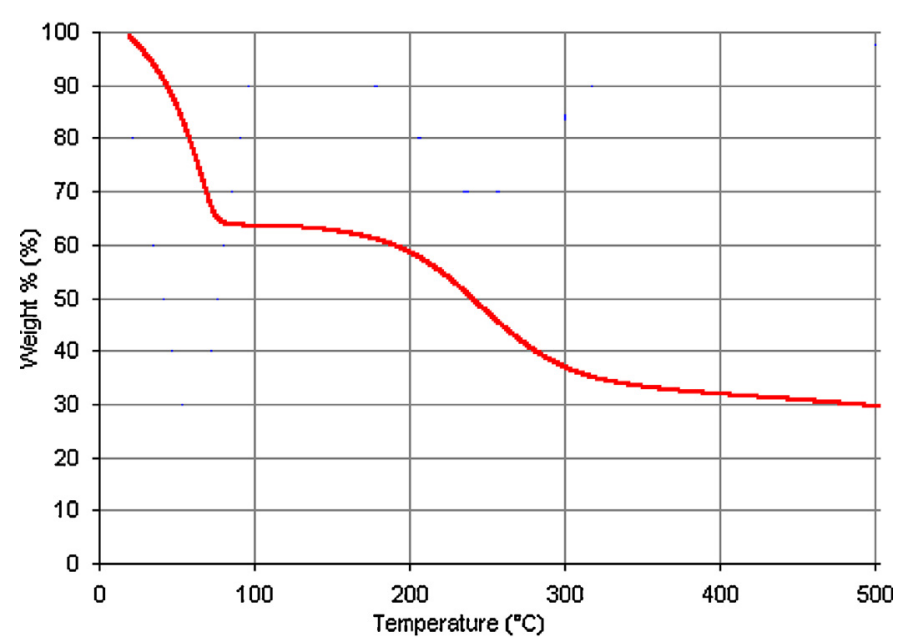

Fig. 2. TGA curves of PPy-DS coating.

attributed to the evaporation of moisture trapped in the pores of the coating. Further loss of weight observed was due to the polymer degradation and desorption temperature was set at $200^{\circ} \mathrm{C}$ in order to maintain reasonable lifetime of the PPy-DS fiber.

Fig. 3 shows the FTIR spectrum of PPy-DS film. The frequencies at 1650 and $1400 \mathrm{~cm}^{-1}$ were attributed to the asymmetric and symmetric pyrrole ring vibration. The peak at $1050 \mathrm{~cm}^{-1}$ was ascribed to in-plane $\mathrm{C}-\mathrm{H}$ stretching vibration and in-plane $\mathrm{N}-\mathrm{H}$ deformation. The bands at $1000 \mathrm{~cm}^{-1}$ reflected the $\mathrm{C}-\mathrm{N}$ stretching vibration and the $=\mathrm{C}-\mathrm{H}$ out-of-plane vibration, which implied the doping state of PPy. The peak at $650 \mathrm{~cm}^{-1}$ can be attributed aromatic $\mathrm{C}-\mathrm{H}$ vibration.

\subsection{GC analysis of pesticides}

Fig. 4 displays typical GC-ECD chromatogram obtained with HSSPME of the pesticide standards using PPy-DS coating. Sufficiently well resolved individual peaks in Gaussian shape were obtained for the pesticides studied with the retention time of 28.3, 28.6, 30.8, 32.0 and 42.6 for ALD, CP, PNZ, PRC, BRP respectively. LMD has given a double peak at a retention time of 44.4 and $44.7 \mathrm{~min}$ and in

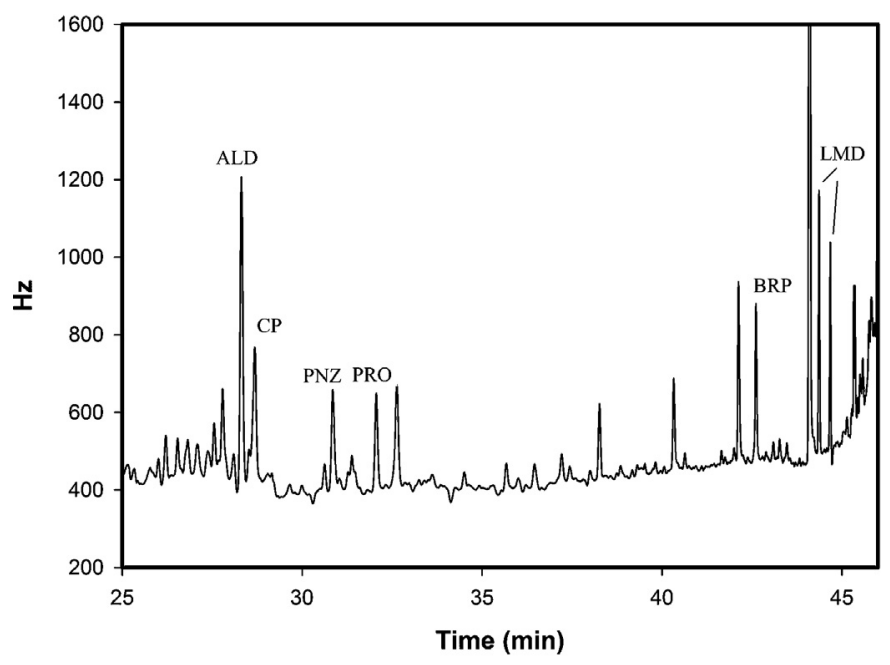

Fig. 4. Chromatograms of ALD, CP, PNZ, PRC, BRP and LMD pesticides injected onto GC column from standard mixture solution in a concentration of $0.05,0.08,20.0$, 20.0, 2.5 and $2.0 \mathrm{ng} \mathrm{mL}^{-1}$, respectively.

the sample analysis the first peak signal was taken into account as much higher signal than the second one was obtained.

\subsection{Optimization of extraction conditions}

The extraction efficiency of the fiber fabricated is strongly dependent on the HS extraction conditions, such as extraction temperature and time, stirring rate and ionic strength of the solution. All experiments are carried out in triplicate and the results were plotted as the peak area ratio of pesticide (As) to that of internal standard (Ai).

\subsection{Extraction temperature}

Volatile components of a sample solution can effectively dissociate from the matrix at elevated temperatures and move into the HS for rapid extraction by the polimeric coating. However, the coating/HS distribution coefficient also decreases with an increasing temperature resulting in a decline in the amount of extracted

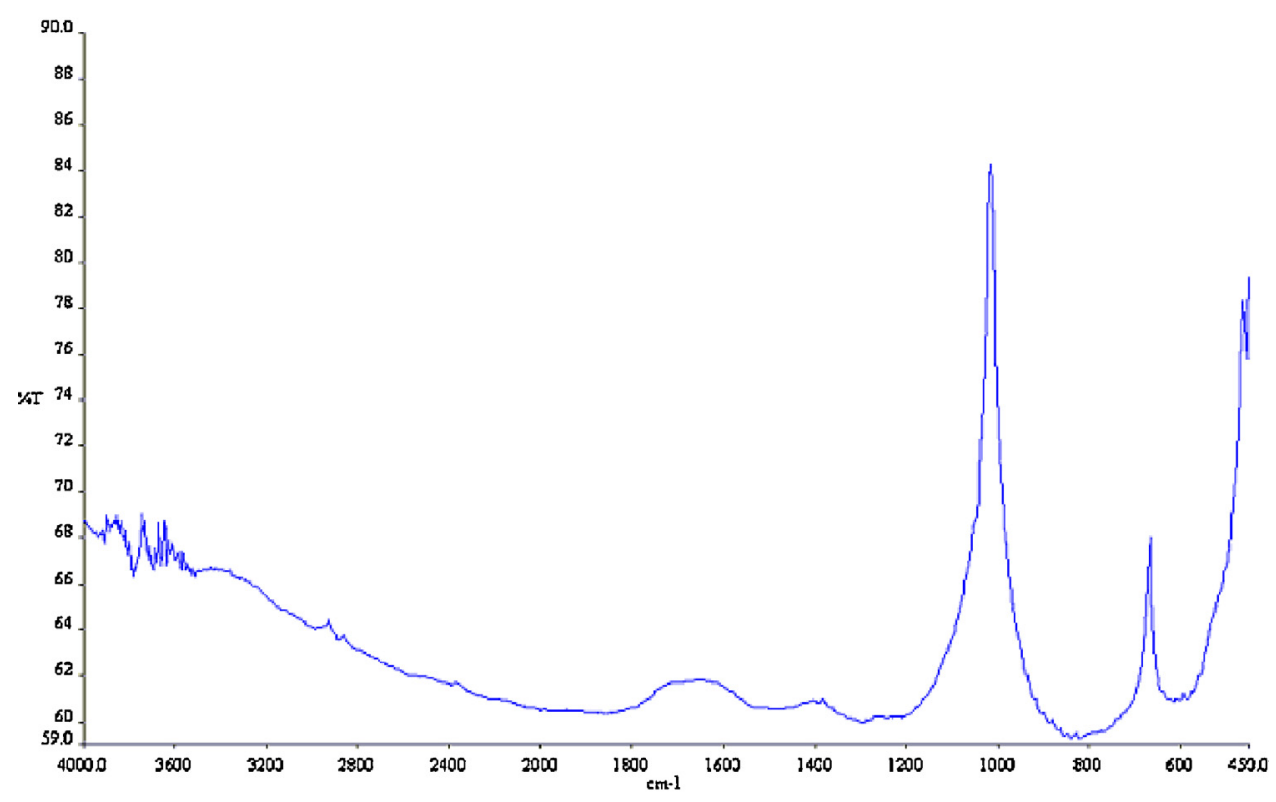

Fig. 3. FTIR spectra of PPy-DS film. 


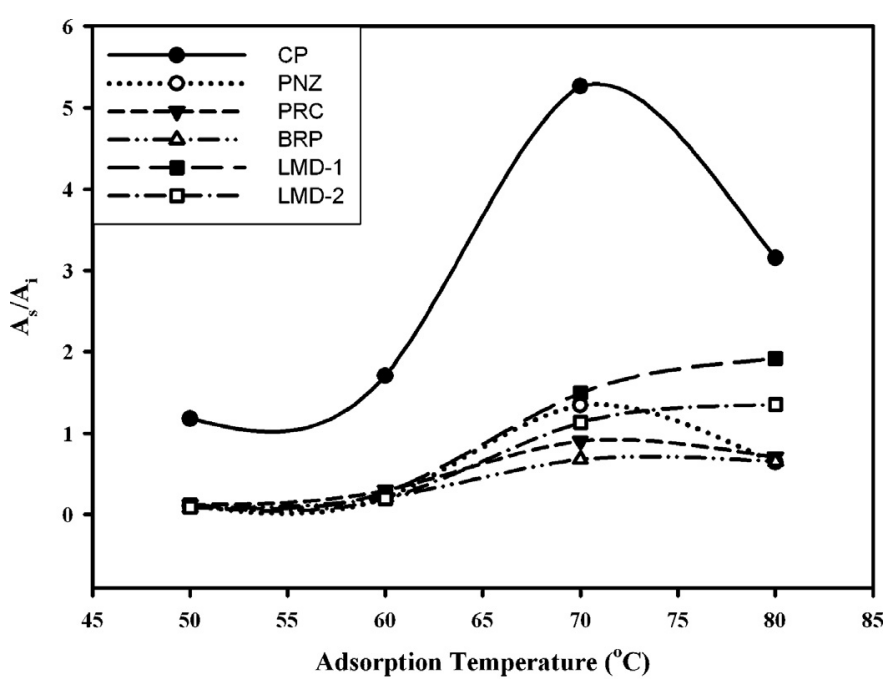

Fig. 5. Effect of extraction temperature on the peak area ratio of As/Ai. Conditions: pesticide mix concentration: $0.8 \mathrm{ng} \mathrm{mL}^{-1} \mathrm{CP}, 150 \mathrm{ng} \mathrm{mL}^{-1} \mathrm{PNZ}, 100 \mathrm{ng} \mathrm{mL}^{-1} \mathrm{PRC}$, $10.0 \mathrm{ng} \mathrm{mL} \mathrm{m}^{-1} \mathrm{BRP}, 10.0 \mathrm{ng} \mathrm{mL}^{-1} \mathrm{LMD}$, sample volume: $10 \mathrm{~mL}, \mathrm{NaCl}: 5 \mathrm{~g} \mathrm{~L}^{-1}$, adsorption time: $15 \mathrm{~min}$, stirring speed $600 \mathrm{rpm}$.

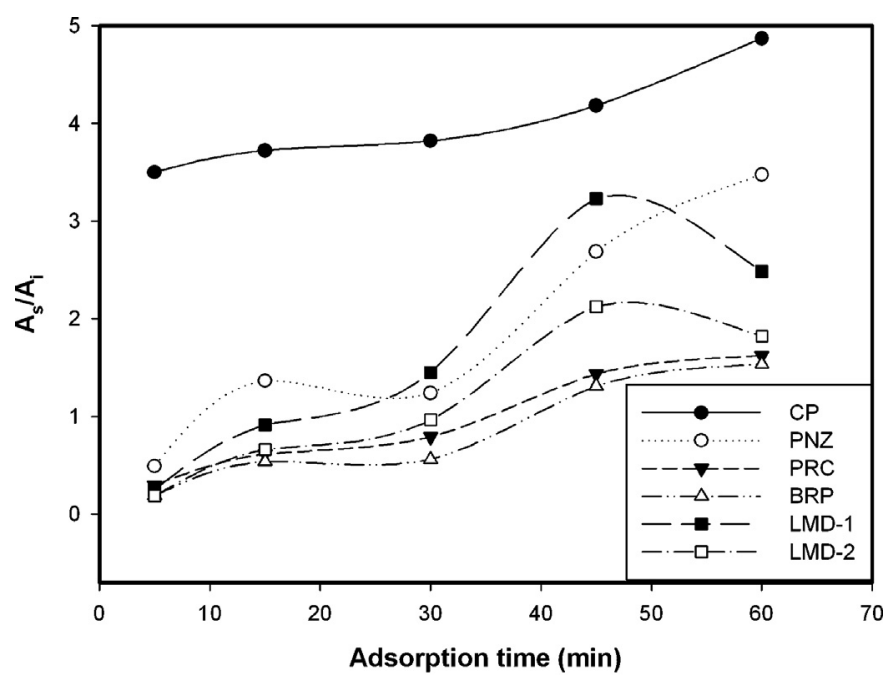

Fig. 6. Effect of extraction time on the peak area ratio of As/Ai. Conditions: pesticide mix concentration: $0.8 \mathrm{ng} \mathrm{mL}^{-1} \mathrm{CP}, 150 \mathrm{ng} \mathrm{mL}^{-1} \mathrm{PNZ}, 100 \mathrm{ng} \mathrm{mL}^{-1} \mathrm{PRC}, 10.0 \mathrm{ng} \mathrm{mL}^{-1}$ $\mathrm{BRP}, 10.0 \mathrm{ng} \mathrm{mL}^{-1} \mathrm{LMD}$, sample volume: $10 \mathrm{~mL}, \mathrm{NaCl}: 10 \mathrm{~g} \mathrm{~L}^{-1}$, adsorption temperature: $70^{\circ} \mathrm{C}$, stirring speed: $600 \mathrm{rpm}$, desorption temperature: $200^{\circ} \mathrm{C}$.

analyte. Therefore, HS temperature should compromise between the two opposite effects. The temperature was changed from 50 to $80^{\circ} \mathrm{C}$ to explore its influence and As/Ai ratios were plotted against the extraction temperature. As shown in Fig. 5, the signal ratios of all pesticides increased with temperature till $70^{\circ} \mathrm{C}$ and then, most signal ratios have decreased at higher degrees, except LMD. Considering the signal loss for some of the pesticides at higher temperatures, the extraction temperature was selected to be as $70^{\circ} \mathrm{C}$.

\subsection{Extraction time}

HS-SPME is an equilibrium-based technique and there is a direct relationship between the amount of the extracted components and the time. Therefore, extraction time designates the sensitivity and reproducibility of the HS SPME. Fig. 6 depicts the extraction time profiles for the studied pesticides from aqueous samples and $45 \mathrm{~min}$ was selected as a reasonable compromise between preconcentration and analysis time.

\subsection{Stirring rate}

It is generally accepted that the reduction of the diffusion layer is essential in order to reach equilibrium faster, which is easily attained by sample agitation. Although the equilibration time progressively decreases with increasing agitation rate, faster agitation tends to be uncontrollable and the rotational speed might cause a change in the equilibration time resulting in poor measurement precision. Fig. 7(A) shows the effect of stirring rate on the peak area ratios for the pesticides studied. As the peak ratios give a maximum at $600 \mathrm{rpm}$, this rate was chosen for further studies.

\subsection{Ionic strength}

For many organic analyte, aqueous solubility decreases with increasing ionic strength, and thus, the partitioning from the aqueous solution to the head space is improved. To raise the ionic strength, an inorganic salt is often added to the aqueous matrix. Fig. 7(B) shows the effect of salt amount on the final signals of the pesticides. Peak areas and peak ratios have given a maximum at $10.0 \mathrm{~g} \mathrm{~L}^{-1} \mathrm{NaCl}$ and this amount of salt was used for further studies.

\subsection{Desorption and conditioning}

For thermal desorption of the pesticides adsorbed on the SPME fiber, a narrow bore ( $1.0 \mathrm{~mm}$ i.d.) unpacked injection liner was used to ensure a high linear carrier gas flow, to reduce desorption time and to prevent peak broadening. Desorption temperature was set at $200^{\circ} \mathrm{C}$ and desorption time was selected as $5 \mathrm{~min}$. However, due to the appearance of a residual signal, fiber surface was conditioned at $200^{\circ} \mathrm{C}$ for $15 \mathrm{~min}$ between two consecutive analyses.

\subsection{Analytical characteristics of the method}

Under optimized conditions, the performance of the method was tested by spiking pesticide standard mixture and a constant amount of internal standard $\left(0.05 \mathrm{ng} \mathrm{mL}^{-1}\right)$ into aqueous ethanol $(12 \% \mathrm{v} / \mathrm{v})$ solution. The correlation coefficients of the calibration curves obtained with and without internal standard were found to be similar and the peak area values alone were used for further studies. Table 1 summarizes the analytical characteristics of the method. The sensitivity of the method was assessed by calculating the detection (LOD) $(\mathrm{S} / \mathrm{N}=3)$ and quantification (LOQ) $(\mathrm{S} / \mathrm{N}=10)$ limits of all pesticides. For the pesticides studied LOD values have followed in the order of $\mathrm{PNZ} \sim \mathrm{PRC}>\mathrm{LMD} \sim \mathrm{BRP}>\mathrm{CP}$ and were calculated as in the range of $0.073-1.659 \mathrm{ng} \mathrm{mL}^{-1}$ with correlation coefficients above 0.98 . Precision of the analysis results was acceptable and low RSD values were obtained for CP and PRC being 2.4\% for $0.08 \mathrm{ng} \mathrm{mL}^{-1}$ and $0.64 \%$ for $4.0 \mathrm{ng} \mathrm{mL}^{-1}$, respectively. However, the RSD \% was found to be higher than $10 \%$ for LMD, PNZ and BRP. The signal consistency for LMD-1 has shown better correlation than that of LMD-2 and therefore, former peak areas were exploited for calibration curves. When the results were compared with recent papers in which were used immersion sampling technique $[48,49]$, lower LOQ results were obtained by this proposed method due to the usage of HS-SPME.

\subsection{Application of the method to the real sample}

In order to verify the applicability of PPy-SPME on to the real samples, the method was applied for Turkish red wine sample. Standard addition method was chosen for analyzing the sample due to the complex matrix effect of red wine. Four standard added samples with different concentration levels with three replicate analyses were performed for all samples. The spiked concentrations for CP were 0.08, 0.12, 0.16, 0.24; for PNZ and PRC were 4.00, 

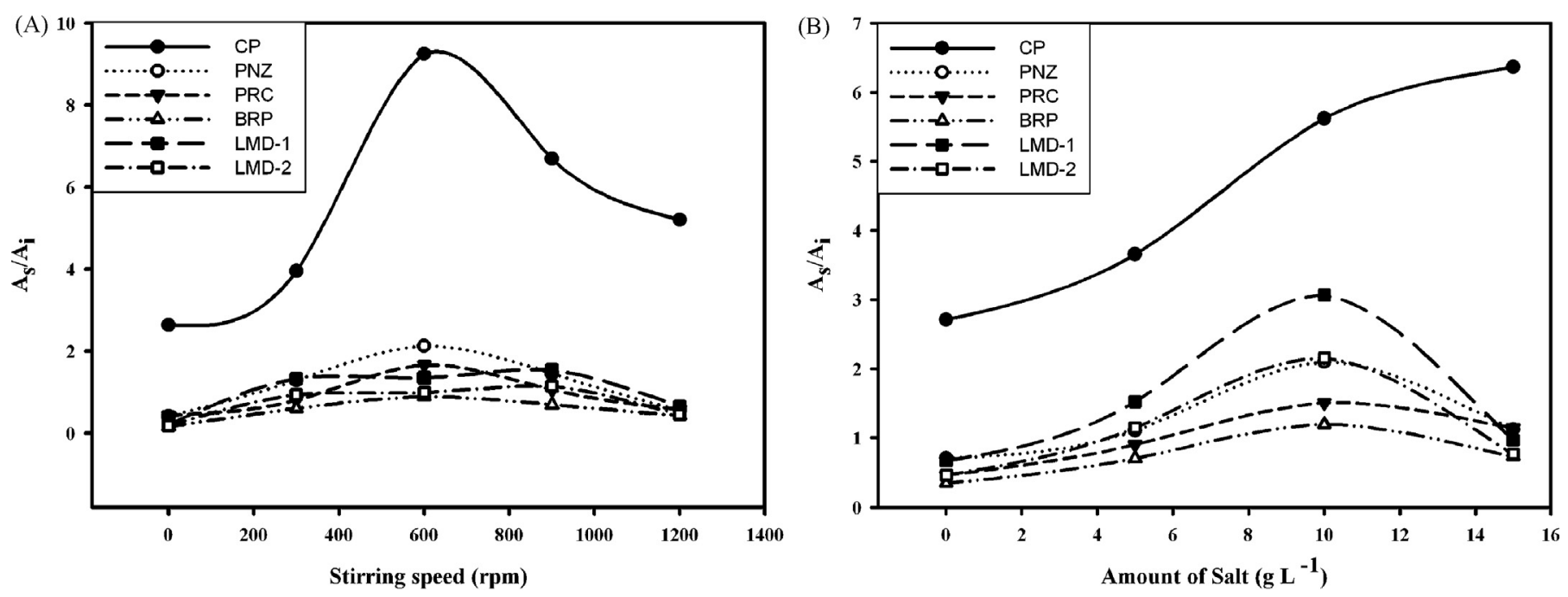

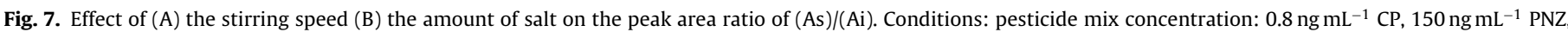

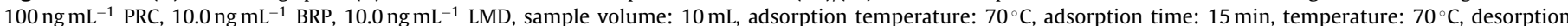
temperature: $200{ }^{\circ} \mathrm{C}$.

Table 1

Analytical characteristics of the proposed method.

\begin{tabular}{|c|c|c|c|c|c|}
\hline Pesticide & Calibration range $\left(\mathrm{ng} \mathrm{mL}^{-1}\right)$ & Equations & $R^{2}$ & LOD ( $\mathrm{ng} \mathrm{mL}^{-1}$ ) & $\mathrm{LOQ}\left(\mathrm{ng} \mathrm{mL}^{-1}\right)$ \\
\hline $\mathrm{CP}$ & $0.08-0.40$ & $y=30,856.684 x+18.406$ & 0.980 & 0.073 & 0.089 \\
\hline PNZ & $4.00-20.00$ & $y=10.461 x+91.402$ & 0.979 & 1.642 & 3.690 \\
\hline PRC & $4.00-20.00$ & $y=51.068 x+72.638$ & 0.984 & 1.659 & 3.289 \\
\hline BRP & $0.50-2.50$ & $y=250.213 x+45.388$ & 0.994 & 0.174 & 0.256 \\
\hline LMD & $0.40-2.00$ & $y=257.871 x+40.267$ & 0.990 & 0.193 & 0.339 \\
\hline
\end{tabular}

Table 2

Recovery results of target pesticides in red wine.

\begin{tabular}{|c|c|c|c|c|c|}
\hline Pesticide & $\mathrm{CP}$ & PNZ & PRC & BRP & LMD \\
\hline$C\left(\mathrm{ng} \mathrm{mL}^{-1}\right)$ & 0.16 & 4.0 & 12.0 & 1.0 & 0.80 \\
\hline Recovery (\%) & $92.2 \pm 14.2$ & $96.1 \pm 25.4$ & $92.7 \pm 14.1$ & $92.9 \pm 6.1$ & $106.8 \pm 10.1$ \\
\hline
\end{tabular}

6.00, 8.00, 12.00; for BRP were 0.50, 0.75, 1.00, 1.50 and for LMD were $0.40,0.60,0.80,1.20 \mathrm{ng} \mathrm{mL}^{-1}$. The target pesticides with in the samples were found to be lower than LOD values. The accuracy of the method was tested upon recovery studies and given in Table 2. As can be followed from the table, high recovery percentages were calculated for all pesticides with acceptable RSD values.

\section{Conclusion}

As a conclusion, present study is the first attempt dealing with the usage of PPy-DS fiber for the HS-SPME determination of endocrine disruptor pesticides in red wine sample. In this study, PPy fiber for HS SPME is fabricated by electrochemical polymerization onto a steel wire and in situ produced fiber coating was proven to have high extraction efficiency for selected pesticides. Although sensitivity of proposed method can be improved by using twisters which can be also applied for HS analysis [52,53], the developed fiber for SPME technique does not require any additional thermal desorption device to ease to desorption of the adsorbed components on the fiber. Gas chromatographic measurements revealed that five endocrine disruptor pesticides those commonly monitored in Turkish red wine sample can be simultaneously detected by the PPy-DS fiber.The method offers an inexpensive alternative for fiber production, offering a reduced cost considerably and improving the selectivity as well as their lifetime. Even after 250 usages, the fiber can still be used sensitively with a reasonable precision. Further studies will cover alternative polymeric matrixes for volatile and semi volatile pesticide analysis in food samples. Although the developed method is mainly focused on the sample pretreatment in particular, separation and preconcentration in a single step, whole procedure can be shortened to $15 \mathrm{~min}$ by employing a fast GC system, as well as a capillary column small in diameter.

\section{Acknowledgement}

The authors thank to the Ege University for financial support (Project 2010/Fen/033).

\section{References}

[1] R. Berlardi, J. Pawliszyn, Water Pollut. Res. J. Can. 24 (1989) 179.

[2] C. Woolley, R. Mindrup, Solid Phase Microextraction: Rapid and Versatile Extraction for GC or HPLC Applications, T296033, vol. 15, No. 3, 19965.

[3] Z. Zhang, J. Pawliszyn, Anal. Chem. 65 (1993) 1843.

[4] C. Dong, W. Wang, Anal. Chim. Acta 562 (2006) 23.

[5] M. Correia, C. Delerue-Matos, A. Alves, J. Chromatogr. A 889 (2000) 59

[6] K. Tsimeli, T.M. Triantis, D. Dimotikali, A. Hiskia, Anal. Chim. Acta 617 (2008) 64.

[7] L. Urruty, M. Montury, M. Braci, J. Fournier, J.M. Dournel, J. Agric. Food Chem. 45 (5) (1997) 1519

[8] J. Martins, C. Esteves, A. Limpo-Faria, P. Barros, N. Ribeiro, T. Simões, M. Correia, C. Delerue-Matos, Anal. Lett. 44 (2011) 1021.

[9] L. Scibetta, L. Campo, R. Mercadante, V. Foà, S. Fustinoni, Anal. Chim. Acta 581 (2007) 53.

[10] J. Martins, C. Esteves, T. Simoes, M. Correia, C. Delerue-Matos, J. Agric. Food Chem. 59 (2011) 6847.

[11] J. Martins, C. Esteves, A. Limpo-Faria, P. Barros, N. Ribeiro, T. Simões, M. Correia, C. Delerue-Matos, Food Chem. 132 (2012) 630. 
[12] R.C. Mejias, R.N. Marin, M.V.G. Moreno, C.G. Barroso, J. Chromatogr. A995 (2003) 11.

[13] F.Q. Guo, L.F. Huang, S.Y. Zhou, T.M. Zhang, Y.Z. Liang, Anal. Chim. Acta 570 (2006) 73.

[14] S. Millan, M.C. Sampedro, N. Unceta, M.A. Goicolea, E. Rodriguez, R.J. Barrio, J. Chromatogr. A 995 (2003) 135.

[15] L.M. Ravelo-Perez, J. Hernandez-Borges, T.M. Borges-Miquel, M.A. RodriguezDelgado, Food Chem. 111 (2008) 764.

[16] M.C.L. Blanco, B.C. Grande, J.S. Gándara, J. Chromatogr. A 963 (2002) 117.

[17] M. Dziadas, H.H. Jeleń, Anal. Chim. Acta 677 (2010) 43.

[18] C.G. Zambonin, M. Quinto, N. De Vietro, F. Palmisano, Food Chem. 86 (2004) 269.

[19] N. Aguinaga, N. Campillo, P. Vinas, M. Hernandez-Cordoba, Spectrosc. Lett. 42 (2009) 320.

[20] S. Risticevic, E. Carasek, J. Pawliszyn, Anal. Chim. Acta 617 (2008) 72.

[21] R.R. Otero, C.Y. Ruiz, B.C. Grande, J.S. Gándara, J. Chromatogr. A 942 (2002) 41.

[22] M.C.L. Blanco, B.R. Rodriguez, B.C. Grande, J.S. Gandara, J. Chromatogr. A 976 (2002) 293.

[23] V.K. Ponnusamy, J.F. Jen, J. Chromatogr. A 1218 (2011) 6861.

[24] H. Bagheri, E. Babanezhad, F. Khalilian, Anal. Chim. Acta 616 (2008) 49

[25] T.V. Vernitskaya, O.N. Efimov, Russian Chem. Rev. 66 (5) (1997) 443.

[26] F. Beck, M. Oberst, R. Jansen, Electrochim. Acta 35 (1990) 1841.

[27] L.X. Wang, X.G. Li, Y.L. Yang, React. Funct. Polym. 47 (2001) 125.

[28] H.S. Nalwa, Handbook of Organic Conductive Molecules and Polymers, WileyVCH, New York, USA, 1997, 3334.

[29] A. Mollahosseini, E. Noroozian, Anal. Chim. Acta 638 (2009) 169.

[30] Y. Meng, V. Pino, J.L. Anderson, Anal. Chim. Acta 687 (2011) 141.

[31] M. Omastova, M. Trchova, J. Kovarova, J. Stejskal, Synth. Met. 138 (2003) 447

[32] A. Mohammadi, Y. Yamini, N. Alizadeh, J. Chromatogr. A 1063 (2005) 1.

[33] N. Alizadeh, H. Zarabadipour, A. Mohammadi, Anal. Chim. Acta 605 (2007) 159.
[34] N. Alizadeh, M. Jafari, A. Mohammadi, J. Hazard. Mater. 169 (2009) 861.

[35] J. Wu, W.M. Mullett, J. Pawliszyn, Anal. Chem. 74 (2002) 4855.

[36] J. Wu, J. Pawliszyn, Anal. Chim. Acta 520 (2004) 257.

[37] J. Pawliszyn, J. Wu, H. Lord, Talanta 54 (2001) 655.

[38] B. Buszewski, P. Olszowy, T. Ligor, M. Szultka, J. Nowaczyk, M. Jaworski, Anal. Bioanal. Chem. 397 (2010) 173.

[39] P. Olszowy, M. Szultka, T. Ligor, J. Nowaczyk, B. Buszewski, J. Chromatogr. B 878 (2010) 2226

[40] M. Szultka, R. Kegler, P. Fuchs, P. Olszowy, W. Miekisch, J.K. Schubert, B. Buszewski, R.G. Mundkowski, Anal. Chim. Acta 667 (2010) 77.

[41] H. Minjia, T. Chao, Z. Qunfang, J. Guibin, J. Chromatogr. A 1048 (2004) 257.

[42] G. Liljegren, L. Nyholm, Analyst 128 (2003) 232.

[43] L. Chen, W. Chen, C. Ma, D. Du, X. Chen, Talanta 84 (2011) 104.

[44] T. Hyötylainen, K. Lüthje, M. Rautiainen-Rama, M.L. Riekkola, J. Chromatogr. A 1056 (2004) 267.

[45] J. Martins, C. Esteves, A.L. Faria, P. Barros, N. Ribeiro, T. Simões, M. Correia, C.D. Matos, Food Chem. 132 (2012) 630

[46] M. Correia, C.D. Matos, A. Alves, Fresenius, J. Anal. Chem. 369 (2001) 647

[47] A. Navalon, A. Prieto, L. Araujo, J.L. Vilchez, J. Chromatogr. A 946 (2002) 239.

[48] A. Navalon, A. Prieto, L. Araujo, L.L. Vilchez, Anal. Bioanal. Chem. 379 (2004) 1100.

[49] R. McKinlay, J.A. Plant, J.N.B. Bell, N. Voulvoulis, Environ. Intern. 34 (2008) 168

[50] M. Zhao, Y. Zhang, W. Liu, C. Xu, L. Wang, J. Gan, Environ. Toxicol. Chem. 27 (5) (2008) 1194

[51] G. Viswanath, S. Chatterjee, S. Dabral, S.R. Nanguneri, G. Divya, P. Roy, J. Steroid. Biochem. Mol. Biol. 120 (1) (2010) 9.

[52] C. Bicchi, C. Cordero, E. Liberto, P. Rubiolo, B. Sgorbini, F. David, P. Sandra, J Chromatogr. A 1094 (2005) 9.

[53] B. Sgorbini, C. Cagliero, C. Cordero, E. Liberto, P. Rubiolo, M.R. Ruosi, C. Bicchi, J. Chromatogr. A 1265 (2012) 39. 